\title{
Lack of association of rare alleles in the HRAS variable number of tandem repeats (VNTR) region with adult glioma ${ }^{1}$
}

\author{
Pengchin Chen, John K. Wiencke, Kathleen Conway, Sharon N. Edmiston, Rei Miike, \\ and Margaret Wrensch ${ }^{2}$ \\ Laboratory for Molecular Epidemiology, Department of Epidemiology and Biostatistics, School of Medicine, \\ University of California, San Francisco, CA $94143-0560$ (P.C., J.K.W., R.M., M.W.); and the Molecular \\ Epidemiology Laboratory, Department of Epidemiology, Lineberger Comprehensive Cancer Center, University \\ of North Carolina, Chapel Hill, NC 27599-7400 (K.C., S.N.E.)
}

HRAS rare alleles have been associated with the increased susceptibility to a variety of cancers. In the present study we examined the hypothesis that HRAS rare alleles are a risk factor for adult glioma in a population-based casecontrol study of adult glioma in six San Francisco Bay Area counties. We compared the prevalence of rare alleles in the variable number of tandem repeats region of HRAS in the germline DNA from 73 white adults who had gliomas with that of 65 controls. Overall, the prevalence of rare alleles in cases was not different from the prevalence of those in controls according to two definitions of rare alleles. We found that 25 of $73(34 \%)$ of cases versus 25 of $65(38 \%)$ of controls had at least one allele that was not $30,46,69$, or 87 repeats; 4 of $73(5 \%)$ of cases versus 6 of $65(9 \%)$ of controls carried one or more alleles with $33,39,42,53,59,63,68,105$, or 114 repeats. The proportion of rare alleles was somewhat higher among subjects with anaplastic astrocytoma. Among women, cases were less likely than controls to have HRAS rare alleles,

Received 3 September 1999, accepted 5 January 2000.

${ }^{1}$ This work was supported by grants CA52689 and CA57220 from the National Cancer Institute; grant IRG-97-150-01-IRG from the American Cancer Society; and Grant ES04705 from the National Institutes for Environmental Health Sciences (NIEHS), through funds provided by the U.S. Environmental Protection Agency (EPA). The results represent the opinions of the authors and not necessarily the official views of the EPA

${ }^{2}$ Address correspondence and reprint requests to Margaret Wrensch, Department of Epidemiology and Biostatistics, University of California, 44 Page St., Suite 503, San Francisco, CA 94143-1215.

${ }^{3}$ Abbreviations used are as follows: $\mathrm{Cl}$, confidence interval; VNTRs, variable number tandem repeats. whereas among men, cases were slightly more likely to have HRAS rare alleles, but none of these results approach statistical significance. Our data do not suggest an excess of HRAS rare alleles among adult glioma cases. Neuro-Oncology 2, 120-124, 2000 (Posted to NeuroOncology [serial online], Doc. 99-46, March 22, 2000. $U R L<$ neuro-oncology.mc.duke.edu>)

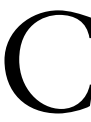

ancer is thought to arise as the result of multiple molecular genetic alterations within tumor cells. These genetic changes fall into two general classes: inactivation of tumor suppressor genes that operate physiologically to restrain growth and activation of proto-oncogenes whose activation can enhance tumor activity. The human HRAS gene is a proto-oncogene homologous to a family of ras viral oncogenes. Activation mutations of HRAS have been implicated in activating a signal transduction pathway that leads to neoplastic transformation of normal cells (reviewed in Barbacid, 1987). In addition to the activation mutations found in human cancer, specific alleles in the HRAS hypervariable minisatellite loci have shown an association with increased susceptibility to a variety of cancers, including bladder, breast, colorectal, and lung cancers, as well as leukemia and melanoma (Krontiris et al., 1993). This minisatellite, located $1 \mathrm{~kb}$ downstream to the HRAS coding sequences, contains a region with VNTRs ${ }^{3}$ of a 28-base pair sequence (Capon et al., 1983). It has been shown that this minisatellite region is capable of binding members of rel/NF-kb transcription regulatory factors (Trepicchio and Krontiris, 1992). The binding of the VNTR region to transcription factors leads to modulation of transcription activation/repression, and the effects on transcription are allele specific (Green and Krontiris, 1993). One study reported a 3 -fold excess of rare HRAS 
alleles in university research hospital patients with diverse histologic types of brain tumors compared with blood donor controls (Diedrich et al., 1988). Gliomas are the most common type of primary brain tumor and account for more than $40 \%$ of all benign and malignant CNS neoplasms. The etiology of adult onset glioma is unknown. Family history and several genes have been implicated in molecular genetic studies. In the present report, we used a polymerase chain reaction approach to study the frequencies of HRAS rare alleles in persons with glioma and controls from a case-control adult glioma study.

\section{Materials and Methods}

The study population was a subgroup of 73 cases and 65 controls from the larger population-based case-control San Francisco Bay Area Adult Glioma Study (Wrensch et al., 1997). The parent study from which these cases and controls were selected included $492(82 \%$ of 603 eligible) incident adult glioma cases (aged $>20$ years) ascertained from January 8, 1991, to March 3, 1994, in 6 San Francisco Bay Area counties through the rapid case ascertainment service of the Northern California Cancer Center. Uniform neuropathology review indicated 4 cases were not glioma, and specimens could not be reviewed for 12 subjects. Thus, the parent study included 476 cases. Controls $(n=462 ; 63 \%$ of those eligible) were contacted through a random digit dialing technique and were frequency-matched for gender and age (within 5 years). We began collecting blood samples partway through the study from 187 cases and 169 controls. The subjects for allelotyping of the HRAS VNTR region represented the first available samples. DNA was extracted from heparinized whole blood, and allelotyping of the HRAS VNTR region was done using a polymerase chain reaction approach as described previously (Conway et al., 1996). Each sample was assigned 2 numbers corresponding to the numbers of 28-base pair repeats between the flanking primers. The assignment of common versus rare alleles is determined by taking into account the combinations of the frequency of alleles in control populations, the size comparison with the previously defined common alleles (a1, a2, a3, and a4), and the sequences of the 28-base pair repeats. The statistical package SAS was used to summarize data and to compute odds ratios and $95 \%$ CIs for rare versus common alleles in cases versus controls (SAS, 1990). Odds ratios and $95 \%$ CIs for comparing cases and controls for rare versus common alleles were computed with the SAS program PROC LOGISTIC. Both unadjusted odds ratios and odds ratios adjusting for age and gender were computed.

\section{Results}

Table 1 compares demographic characteristics and tumor histology of cases in the study group with the overall parent study population. Only whites, for both cases and controls, were included in the HRAS VNTR study group because distribution of HRAS allelotypes are highly ethnicity dependent (Weston et al., 1991), and $84 \%$ of people in the Glioma Study are white. The HRAS VNTR study group was younger than the overall study population. This was true for cases (48.6 years old versus 54.2 years old) and controls (49.1 years old versus 53.7 years old). The percentages of astrocytic gliomas in the parent and this substudy were comparable $(88 \%$ versus $84 \%)$. However, the percentage of glioblastomas in the HRAS VNTR study group was lower than in the parent study population.

The distributions of specific HRAS VNTR alleles and allelotypes in cases and controls are shown in Fig. 1. For 3 cases, molecular analysis was insufficient to resolve both alleles, but indicated presence of at least one 30repeat allele. These 3 cases are not shown in Fig. 1. Over-

Table 1. The study group included in HRAS rare allele analysis compared with the overall study population of brain tumor cases and controls in the San Francisco Bay Area Adult Glioma Study, 1991-1995

\begin{tabular}{|c|c|c|c|c|}
\hline \multirow[b]{2}{*}{ Factor } & \multicolumn{2}{|c|}{ Total study population } & \multicolumn{2}{|c|}{ HRAS analyses population subset } \\
\hline & Cases $(n=476)$ & Controls $(n=462)$ & Cases $(n=73)$ & Controls $(n=65)$ \\
\hline \multicolumn{5}{|l|}{ Sex } \\
\hline Female (\%) & 43 & 45 & 40 & 43 \\
\hline Male (\%) & 57 & 55 & 60 & 57 \\
\hline Age (years) (mean $\pm \mathrm{SE}$ ) & $54.2 \pm 0.8$ & $53.7 \pm 0.8$ & $48.6 \pm 1.8$ & $49.1 \pm 1.9$ \\
\hline \multicolumn{5}{|l|}{ Diagnosis by cell type: $(\%)^{\mathrm{a}}$} \\
\hline All astrocytic tumors & $417(88)$ & & $61(84)$ & \\
\hline Glioblastoma & $281(59)$ & & $32(44)$ & \\
\hline Anaplastic astrocytoma & $63(13)$ & & $9(12)$ & \\
\hline Astrocytoma & $26(6)$ & & $5(7)$ & \\
\hline Oligoastrocytoma & $47(10)$ & & $15(21)$ & \\
\hline Others & $59(12)$ & & $12(16)$ & \\
\hline Race (white) (\%) & 84 & 86 & 100 & 100 \\
\hline
\end{tabular}

The study group included whites only.

${ }^{a}$ Numbers in parentheses are percent of total tumors. 


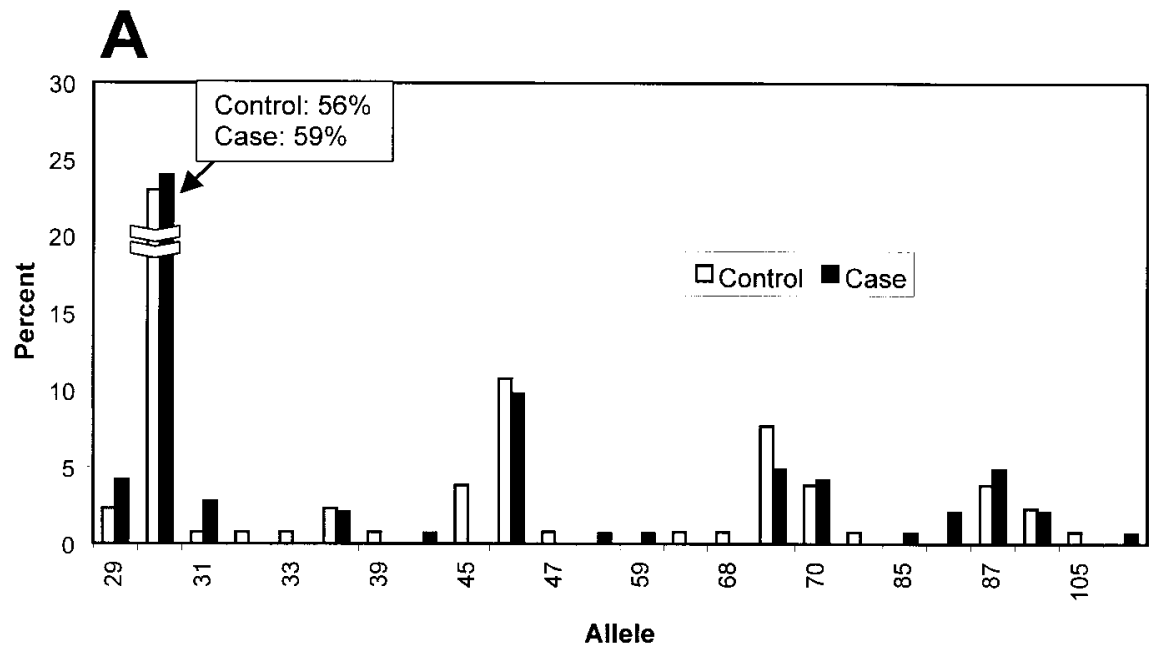

B

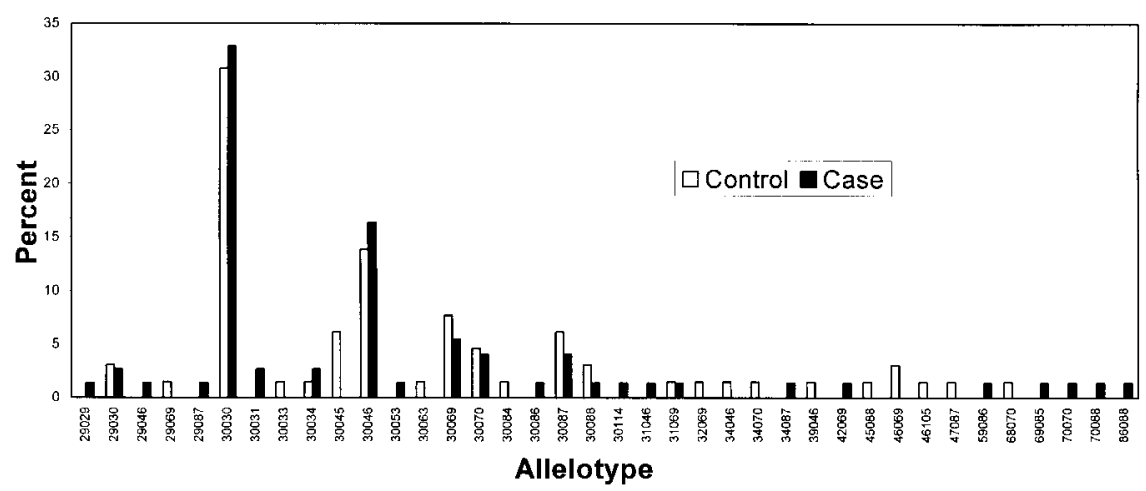

Fig. 1. Percent distribution of HRAS variable number of tandem repeat (VNTR) alleles and allelotypes for adult glioma cases and controls. A. Percent distribution of individual HRAS VNTR alleles. Numbers on the allele axis represent numbers of 28-base pair repeats in each allele. B. Percent distribution of HRAS VNTR allelotypes. Numbers on the allelotype axis represent combined allelotypes for each subject. The first two digits represent the number of repeats for the alleles with fewer repeats, and the last three digits represent the number of repeats for the alleles with more repeats. Open bars and closed bars represent percentages of controls and cases with specific allelotypes, respectively.

all, the prevalence of rare alleles in cases was not different from the prevalence in controls using two definitions of rare alleles. We found 25 of $73(34 \%)$ of cases versus 25 of $65(38 \%)$ of controls had at least one allele that was not $30,46,69$, or 87 repeats; 4 of $73(5 \%)$ of cases versus 6 of $65(9 \%)$ of controls carried one or more alleles of $33,39,42,53,59,63,68,105$, or 114 repeats (Table 2). Table 2 shows the prevalence of the HRAS rare alleles according to both definitions of rare by histologic type. Odds ratios are $0.83(95 \% \mathrm{CI}=0.4-1.7)$ for cases to have at least one allele that was not $30,46,69$, or 87 repeats and $0.57(95 \% \mathrm{CI}=0.2-2.1)$ for cases to carry one or more alleles of $33,39,42,53,59,63,68,105$, or 114 repeats. These odds ratios and CIs were altered only slightly by adjustment for age and sex. These results do not suggest an excess of HRAS rare alleles among adult glioma cases. The proportion of rare alleles was somewhat higher among anaplastic astrocytic cases, but these results are not statistically significant. Table 3 shows that in women cases were less likely than controls to have $H R A S$ rare alleles, while in men cases were slightly more likely to have HRAS rare alleles.
Table 2. Frequencies of HRAS rare alleles in white brain tumor patients and controls, stratified by tumor histopathology, in the San Francisco Bay Area Adult Glioma Study, 1991-1995

\begin{tabular}{lcc} 
& \multicolumn{2}{c}{ HRAS allelotypes } \\
\cline { 2 - 3 } Group & No. rare $(\%)^{\mathrm{a}}$ & No. rare $(\%)^{\mathrm{b}}$ \\
\hline Control $(n=65)$ & $25(38)$ & $6(9)$ \\
All cases $(n=73)$ & $25(34)$ & $4(5)$ \\
Glioblastoma $(n=32)$ & $12(38)$ & $2(6)$ \\
Anaplastic astrocytoma $(n=9)$ & $5(55)$ & $0(0)$ \\
Astrocytoma $(n=5)$ & $0(0)$ & $0(0)$ \\
Oligodendroglioma $(n=7)$ & $1(14)$ & $0(0)$ \\
Oligoastrocytoma $(n=15)$ & $5(33)$ & $2(13)$ \\
Juvenile pilocytic astrocytoma $(n=3)$ & $1(33)$ & $0(0)$ \\
Ependymoma $(n=2)$ & $1(50)$ & $0(0)$ \\
\hline
\end{tabular}

${ }^{a}$ Number and percentage of subjects who carry one or more rare alleles according to previously defined four common alleles by Southern blotting analyses. Alleles with 30, 46, 69, or 87 repeats corresponded to common alleles a1, a2, a3, and a4, respectively; all else are rare alleles.

${ }^{b}$ Number and percentage of subjects who carry one or more rare alleles according to Conway's (K.C.) definition: 19, 33, 39, 42, 53, 59, 63, 68, 84, 105, and 114 repeats are rare; all else are common. 
Table 3. Frequencies of HRAS rare alleles in white brain tumor patients and controls, stratified by sex, in the San Francisco Bay Area Adult Glioma Study, 1991-1995

\begin{tabular}{|c|c|c|c|c|}
\hline \multirow[b]{2}{*}{ HRAS allelotypes } & \multicolumn{2}{|c|}{ Females } & \multicolumn{2}{|c|}{ Males } \\
\hline & Control $(n=28)$ & Case $(n=29)$ & Control $(n=37)$ & Case $(n=44)$ \\
\hline Rare, defined previously ${ }^{\mathrm{a}}(\%)$ & $14(50)$ & $9(31)$ & $11(30)$ & $16(36)$ \\
\hline Rare, defined by Conway $(\%)$ & $3(11)$ & $0(0)$ & $3(8)$ & $4(9)$ \\
\hline
\end{tabular}

${ }^{a}$ Number and percentage of subjects who carry one or more rare alleles according to previously defined four common alleles by Southern blotting analyses. Alleles with $30,46,69$, or 87 repeats corresponding to common alleles a1, a2, a3, and a4, respectively; all else are rare alleles.

${ }^{b}$ Number and percentage of subjects who carry one or more rare alleles according to Conway's (K.C.) definition: 33, 39, 42, 53, 59, 63, 68, 84, 105, and 114 repeats are rare; all else are common.

\section{Discussion}

Although a wide variety of cancers have been investigated for an association with rare HRAS alleles, results have been inconclusive. Only one other study examined the relationship between HRAS rare alleles and the occurrence of brain tumors (Diedrich et al., 1988). This study found higher incidence of rare hypervariable HRAS alleles in intracranial tumor patients in comparison with healthy blood donors. We did not observe an increased risk for adult glioma in individuals with HRAS rare alleles. There are several differences in the studies that might contribute to the different conclusions reached. First, brain tumors included in the previous study consisted of several heterogeneous histopathologic types of cancers that were not included in our study (for example, meningioma). Cytogenetic and molecular genetic analyses of glioma indicate substantial genetic heterogeneity even within and between histologic grades of gliomas (von Deimling et al., 1995). Thus, different groupings and classifications of brain tumors may have affected the study outcomes. Secondly, in the present study, samples were drawn from glioma cases and controls frequency-matched to cases by gender and age from a population-based epidemiologic study, whereas the other study compared a case series from an academic hospital matched with healthy blood-donor controls. We also took into consideration potential effects of ethnicity on HRAS allelotypes by including only whites in this allelotyping study. In addition, the molecular assays employed in the two studies were different. Our approach of using polymerase chain reaction-based assays results in higher resolution of VNTR fragments and thus increases the number of alleles detected for the HRAS VNTR region. Finally, it should be noted that, although our study was negative, it had $80 \%$ power to detect odds ratios of 2.8 for rare allele definition one and 4.2 for rare allele definition two. The previous study indicated an odds ratio of approximately 4 for gliomas, a magnitude of risk our study should have been able to detect. Thus, we were not able to confirm an association of rare $H R A S$ alleles with glioma risk.

Cases in this substudy of HRAS VNTR tended to be younger and have lower grade tumors than all population-based cases, because blood samples were not collected until (at times up to 6 months) after the interview. Thus, since younger patients and patients with lower grades of brain tumors have better survival, they are overrepresented in this sample. This difference was primarily due to underrepresentation of the glioblastoma group, the histologic type of glioma with the poorest survival. Although the percentage of glioblastoma cases in the HRAS VNTR study group was lower than that of the parent study population ( $44 \%$ versus $59 \%$, Table 1$)$, the percentages of anaplastic astrocytoma cases were similar between the HRAS VNTR study group and the parent study population $(12 \%$ versus $13 \%$, Table 1$)$. Another factor that might contribute to finding no association of HRAS VNTR rare alleles and glioblastoma is the fact that glioblastomas are highly heterogeneous, genetically. There is increasing evidence that glioblastoma consists of several distinct entities with different genetic alterations (Louis, 1997; Watanabe et al., 1996).

Because we found a somewhat positive association of $H R A S$ rare alleles with a small sample of subjects with anaplastic astrocytoma, and because others have reported overexpression of HRAS in high-grade astrocytoma tumors (Orian et al., 1992; Riccardi et al., 1991), it may be worthwhile to follow-up with a study to determine if the HRAS rare alleles are associated with anaplastic astrocytoma in other populations. Given that most high-grade astrocytomas that were found in other studies to have elevated HRAS protein expression were glioblastomas, and glioblastomas themselves represent a highly heterogeneous group of tumors, it might also be of interest to extend the studies to subsets of glioblastoma that have a genetic profile similar to anaplastic astrocytoma.

\section{References}

Barbacid, M. (1987) ras genes. Ann. Rev. Biochem. 56, 779-827.

Capon, D.J., Chen, E.Y., Levinson, A.D., Seeburg, P.H., and Goeddel, D.V.

(1983) Complete nucleotide sequences of the T24 human bladder carci-

noma oncogene and its normal homologue. Nature 302, 33-37.
Conway, K., Edmiston, S.N., Hulka, B.S., Garrett, P.A., and Liu, E.T. (1996) Internal sequence variations in the $\mathrm{Ha}$-ras variable number tandem repeat rare and common alleles identified by minisatellite variant repeat polymerase chain reaction. Cancer Res. 56, 4773-4777. 
Diedrich, U., Eckermann, O., and Schmidtke, J. (1988) Rare Ha-ras and c-mos alleles in patients with intracranial tumors. Neurology 38, 587-589.

Green, M., and Krontiris, T.G. (1993) Allelic variation of reporter gene activation by the HRAS1 minisatellite. Genomics 17, 429-434.

Krontiris, T.G., Devlin, B., Karp, D.D., Robert, N.J., and Risch, N. (1993) An association between the risk of cancer and mutations in the HRAS1 minisatellite locus. N. Engl. J. Med. 329, 517-523.

Louis, D.N. (1997) A molecular genetic model of astrocytoma histopathology. Brain Pathol. 7, 755-764.

Orian, J.M., Vasilopoulos, K., Yoshida, S., Kaye, A.H., Chow, C.W., and Gonzales, M.F. (1992) Overexpression of multiple oncogenes related to histological grade of astrocytic glioma. Br. J. Cancer 66, 106-112.

Riccardi, A., Danova, M., Giordano, M., Gaetani, P., Butti, G., Zibera, C., and Mazzini, G. (1991) Proto-oncogene expression and proliferative activity in human malignant gliomas. Dev. Oncol. 66, 81-84.

SAS (1990) Vol. 2. Cary, N.C.: SAS Institute, Inc.
Trepicchio, W.L., and Krontiris, T.G. (1992) Members of the rel/NF-kappa B family of transcriptional regulatory proteins bind the HRAS1 minisatellite DNA sequence. Nucleic Acids Res. 20, 2427-2434.

von Deimling, A., Louis, D.N., and Wiestler, O.D. (1995) Molecular pathways in the formation of gliomas. Glia 15, 328-338.

Watanabe, K., Tachibana, O., Sata, K., Yonekawa, Y., Kleihues, P., and Ohgaki, H. (1996) Overexpression of the EGF receptor and p53 mutations are mutually exclusive in the evolution of primary and secondary glioblastomas. Brain Pathol. 6, 217-224.

Weston, A., Vineis, P., Caporaso, N.E., Krontiris, T.G., Lonergan, J.A., and Sugimura, H. (1991) Racial variation in the distribution of Ha-ras-1 alleles. Mol. Carcinog. 4, 265-268.

Wrensch, M., Lee, M., Miike, R., Newman, B., Barger, G., Davis, R., Wiencke, J., and Neuhaus, J. (1997) Familial and personal medical history of cancer and nervous system conditions among adults with glioma and controls. Am. J. Epidemiol. 145, 581-593. 